\title{
VARIABLES ANTROPOMÉTRICAS Y SU RELACIÓN CON LA FUERZA-PRENSIÓN DE MANO, PARA EL USO ERGONÓMICO DE HERRAMIENTAS MANUALES EN UN GRUPO DE TRABAJADORES DEL SECTOR DE LA CONSTRUCCIÓN EN BOGOTÁ
}

\author{
Anthropometric variables and its relationship with the \\ force - hand grip, for ergonomic use of hand tools in \\ a group of workers in the field of building at Bogota
}

ALDO PIÑEDA GERALDO*, LADY CABRERA OSORIO**, CAROLINA ESGUERRA SABOGAL**, JUAN GRAJALES CORREA**, CRISTHIAN GONZÁLEZ**

Recibido: 20 de Noviembre de 2015. Aceptado: 30 de Noviembre de 2015

DOI: http://dx.doi.org/10.21017/rimci.2016.v3.n5.a7

\begin{abstract}
Resumen
El presente artículo tuvo como objetivo evaluar la relación entre las medidas antropométricas y la fuerza prensil de las manos para el uso de herramientas manuales. El estudio fue descriptivo ya que se detallaron algunas medidas de las manos y la fuerza prensil. Los participantes fueron un grupo de trabajadores del sector de la construcción en Bogotá y se utilizaron los siguientes instrumentos de medición: un vernier de ramas cortas de 0 a 200 mm, una cinta métrica y un dinamómetro hidráulico de mano, para la medición de la fuerza prensión digito-palmar. Todos los datos recopilados se registraron en una ficha individual por trabajador. En cuanto a las medidas de la mano, se evidenció que las fuerzas están en el dedo índice y el dedo medio, a mayor dimensión de los dedos mayor fuerza. Los trabajadores deben usar herramientas ergonómicas para prevenir riesgos laborales ya muchas de las veces no están adaptada para la mano.
\end{abstract}

Palabras clave: antropometría, fuerza, prensión, mano, herramientas manuales.

\begin{abstract}
This article aimed to evaluate the relationship between anthropometric measures and the grip strength of hands using hand tools. The study was descriptive as some measures of hands and grip strength were detailed. The participants were a group of workers in the construction sector in Bogota and the following measuring instruments were used: a vernier of short branches from 0 to $200 \mathrm{~mm}$, a tape measure and a hydraulic hand dynamometer for measuring force finger-palmar grasp. All collected data were recorded in a single tab per worker. As for hand measurements, it was evident that the forces are on the index finger and middle finger, a larger force of greater fingers. Workers should use ergonomic tools to prevent occupational hazards and many times are not adapted to the hand.
\end{abstract}

Keywords: anthropometry, strength, seizing, hand, tool hand made.

\footnotetext{
* Antropólogo Físico de la Escuela Nacional de Antropología e Historia de México, D.F. Docente-Investigador grupo O.C.A de la Corporación Universitaria Republicana. Correo electrónico: apineda@docente.urepublicana.edu.co

* Estudiante de la Facultad de Ingeniería Industrial de la Corporación Universitaria Republicana.
} 


\section{INTRODUCCIÓN}


trucción se presenta por los altos riesgos de lesiones músculo-esqueléticos relacionados con las tareas de manos y muñecas. Asimismo, se presenta entre los trabajadores una alta demanda física de posturas forzadas y el uso frecuente de herramientas manuales. Por el uso inadecuado de las herramientas manuales, se presentan problemas físicos como: lesiones, golpes, movimientos bruscos, movimientos repetitivos, fatiga y cansancio de las manos, así mismo las herramientas con frecuencia no son las adecuadas, ya que no están diseñada para las tareas y para la población específica de este sector. El presente estudio tuvo como objetivos: evaluar la relación entre las medidas antropométricas y la fuerza prensil de las manos, para el buen uso de herramientas manuales. Otro de los objetivos fue calcular los valores de la fuerza de prensión a través de la dinamometría.

\section{Problema}

Uno de los principales problemas del sector de la construcción son los riesgos de lesiones músculos esqueléticas relacionados con el trabajo de las manos y muñecas. También, se presenta entre los trabajadores una alta demanda física por posturas forzadas y el uso frecuente de herramientas manuales.

Por otro lado, el uso inadecuado de las herramientas manuales, provocan problemas físicos como son: las lesiones por golpes, movimientos repetitivos, fatiga y cansancio de las manos. Las herramientas no son las adecuadas, ya que no están diseñadas para las tareas y para la población específica de este sector productivo. El objetivo fue la identificación y evaluación de las variables antropométricas de la mano derecha y fuerzas de prensión, para ajustar el uso de herramientas manuales y así mejorar la productividad de este grupo de trabajadores del sector de la construcción en Bogotá, Distrito Capital.

Al trabajar con una herramienta manual funcionan pequeños grupos musculares, si se da un sobreesfuerzo estos se exponen a una fatiga a corto plazo. Esto no se percibe fácilmente por lo que pueda resultar sobreexpuesto. Así mismo, otras estructuras como los tendones, inserciones, nervios, también pueden verse afectadas [1]. Por otro lado, Cerda, Cubillos y Medina explican que una inadecuada relación entre persona/herramienta y el uso inadecuado de la misma, puede ser un riesgo de traumas músculo-esquelético de la mano [2].

Mondelo, describe algunos factores que pueden desencadenar problemas músculos esqueléticos como son [3]: la herramienta no es la adecuada para desempeñar una actividad propuesta, la herramienta no está proporcionalmente diseñada para ejercer una tarea y las herramientas no fueron diseñadas para la población específica. Además «Desde el punto de vista técnico, para el correcto diseño de herramientas en base a una población determinada, debemos considerar medidas antropométricas de la mano vinculadas directamente con medidas dimensionales del objeto diseñado»[2].

Existe evidencia que los desórdenes músculo esqueléticos son los principales problemas en la industria de la construcción [4], debido a que las actividades que se realizan en este tipo de industria comúnmente requieren elevada demanda física de trabajo, posturas forzadas, levantamiento de cargas, uso frecuente de herramientas manuales y otros esfuerzos enérgicos [5]. Se ha demostrado que los trabajadores de la construcción muestran elevado riesgo de desarrollar lesiones músculo esqueléticas relacionadas con el trabajo en espalda baja, hombros, manos, muñecas y rodillas, lo cual genera elevados costos para las empresas y la fuerza de trabajo [5-7].

Las herramientas manuales juegan un papel muy importante, ya que es la relación entre el trabajador y el proceso productivo realizado. Son muchas las variables que entran en juego, pero el resultado cuando no se realiza una adaptación al trabajador puede ser la producción de lesiones de diversas índoles. En las innovaciones tecnológicas de las herramientas manuales y guantes, se deben hacer mejoras para asegurar la interface entre éstas y el operador y que sean de un mejor uso y más óptima, lo que esta redundará en mejores condiciones laborales y de bienestar.

El mal diseño, fabricación y uso inadecuado de las herramientas manuales provocan problemas físicos que se manifiestan en accidentes, lesiones, golpes, movimientos repetitivos (micro 
traumatismos), fatiga y cansancio de las manos. En el diseño, selección y uso de herramientas, debemos tener parámetros a evaluar si queremos obtener un sistema ergonómico productivo óptimo. Al hacer uso de las herramientas manuales se debería exigir un análisis ergonómico y de esta manera las manos, brazo y antebrazo de los operarios será beneficiadas con lo cual se mostrará una reducción de presiones a ejercer, igualmente se pueden modificar mangos más anatómicos de acuerdo a la medidas de las manos y adaptarlos al tipo de tareas y actividades [3].

A nuestro modo de ver es importante proteger las manos no solamente de los esfuerzos y de las exposiciones a movimientos repetitivos, también es de considerable importancia el protegerlas de los riesgos que se presentan al no utilizar elementos de protección personal adecuados para la labor que se está desarrollando. Para proteger al trabajador de todos los riesgos que se generan al manipular herramientas o materiales filosos, bordes cortantes, virutas metálicas, ciertos golpes y otros tantos riesgos físicos, hay que adoptar las medidas adecuadas de higiene y seguridad en el trabajo, para la prevención de accidentes. Para el caso particular de proteger las manos de los riesgos descritos existen distintos tipos de guantes confeccionados en diferentes materiales que se ajustan a exigentes Normas de Higiene y Seguridad en el Trabajo.

Basándonos en la norma OHSAS 18001:2007, donde se debe propender por identificar y evaluar los riesgos que se presentan en el entorno laboral y de esta manera poder establecer controles para reducirlos a su mínima expresión, muchas empresas buscan certificarse o cumplir con la norma como estrategia de gestión, es en este punto donde un análisis del uso actual de los guantes como elemento de protección de la mano y como extensión de la misma en el momento que se interactúa con las herramientas manuales, este estudio abordó las siguientes preguntas:

1) ¿Cuáles son las características antropométricas y la relación con la fuerza de la mano, para el buen uso ergonómicos de herramientas manuales?

2) ¿Son adecuados los guantes para el tipo de herramienta que se usa?
3) ¿El guante permite el correcto desarrollo de la fuerza de prensión que se está aplicando?

Estas variables se deben tener en cuenta cuando se realiza el estudio de las fuerzas de prensión y el buen uso de las herramientas manuales.

\section{Anatomía de la mano}

Es completamente claro que la disposición anatómica de la mano es lo que le permite al hombre tener gran variedad de adaptaciones funcionales en un momento determinado de acuerdo a la necesidad. Constantemente la mano asume formas diversas que le permiten al hombre interactuar con el medio externo; posiciones como la concavidad palmar que permite tomar y soltar objetos, movimientos de oposición que proporcionan la pinza y facilitan la manipulación de instrumentos de precisión y actividades de destreza fina.

La disposición anatómica de la mano nos permite comprender la gran versatilidad que se puede lograr en la manipulación de objetos y ajustes posicionales de acuerdo a las necesidades en la ejecución de ciertos patrones funcionales. La función prensil de la mano depende de la integridad de la cadena cinética de huesos y articulaciones extendida desde la muñeca hasta las falanges distales y la ruptura de alguna de ellas compromete el ensamblaje necesario para la realización de agarres de fuerza y de precisión. Las unidades adaptativas de la mano que se mueven alrededor son tres elementos: el pulgar, el índice y la unión del tercero, juntos con el cuarto y quinto metacarpianos [8].

\section{A. Antropometría y medidas de las manos}

La antropometría se ha definido de acuerdo a sus intereses y área de estudios. En el diccionario de la Lengua Española se definió así: Antropometría. (De antropo- y -metría). f. Tratado de las proporciones y medidas del cuerpo humano [9]. «La antropometría está relacionada con las dimensiones y otras características del cuerpo; tales medidas están, por supuesto relacionadas con el diseño de las cosas que las personas usan» [10]. La antropometría es una técnica que estudia las dimensiones del cuerpo humano, lo mismo con objetivos antropológicos, médicos, deportivos, que para el diseño de sistemas de 
los que la persona forma parte: objetos, herramientas, muebles, espacios y puestos de trabajo. La diferencia estriba precisamente en los objetivos con que se utilice [3].

La antropometría utiliza la ergonomía como la ciencia encargada de estudiar tanto las dimensiones del ser humano incluidos sus movimientos, como su peso, su volumen, sus fuerzas, sus desplazamientos angulares [3].

\section{1) La longitud de la mano (LM)}

El antebrazo y la palma de la mano deberán estar extendidos, apoyadas sobre una superficie plana y cómoda y los dedos unidos, se mide la distancia entre los puntos antropométricos estilión y dactilión. Esta medida se toma con el calibrador de ramas cortas. Con el punto dactilión que es el punto más distal del dedo medio (tercer dedo), podemos determinar la altura y la longitud de la mano, ya sea la mano derecha e izquierda. Esta medida puede ser útil para el diseño de guantes, la muñeca es el punto de referencia para el espacio del movimiento de la mano.

\section{2) Longitud de la palma de la mano (LPM)}

Esta medida se toma con el compás de ramas cortas o vernier, es la distancia entre el pliegue de la piel, más próxima de la muñeca, entre el punto más distal de la muñeca hasta la articulación del metacarpofalange del dedo medio.

\section{3) Anchura máxima de la palma de la mano AMPM)}

Se mide con el calibrador de ramas cortas o vernier, es la distancia entre los planos más laterales de la palma, perpendiculares al eje de la mano, cuidando de no comprimir los tejidos. Es un indicador del espacio de movimientos lateral de la mano.

\section{4) Anchura máxima de la mano con el pulgar (AMMP)}

Se mide con el calibrador de ramas cortas o vernier, es la distancia del plano cubital de la palma al borde más lateral del pulgar, el dedo debe estar unido suavemente a la palma. Se puede utilizar para el diseño de guantes y otros elementos de protección para las manos.

\section{5) Espesor de la mano (EM)}

Se mide con el calibrador de ramas cortas o el vernier, es la medida del espesor máximo de la mano, medido sobre los nudillos. Se puede utilizar para el diseño de guantes $u$ otros elementos de protección para las manos.

\section{6) Diámetro de empuñadura (DE)}

Para tomar esta medida se utiliza un cono de medición y se le pide a la persona que una su pulgar e índice y recorra el cono hasta encontrar el diámetro que le permita mantener los dedos juntos, con suavidad. Se mide el diámetro del cono en ese pulgar. Se aplica para calcular el diámetro cómodo de confort para el uso de palancas, asas y otros usos de instrumentos de trabajo con las manos [11].

De acuerdo a Vallois, se puede utilizar el índice de la mano siendo la anchura de la mano por cien, dividido entre la longitud de la mano. La clasificación que hace referencia el autor es el siguiente: mano pequeña, X-42.9, mano mediana de 43 a 47.9 y mano grande 48 en adelante [12].

Anchura de la mano x 100 Longitud de la mano

Tabla 1. Clasificación

\begin{tabular}{lc}
\hline Mano pequeña & $X-42.9$ \\
Mano mediana & 43 a 47.9 \\
Mano grande & $>$ de 48 \\
\hline
\end{tabular}

Fuente: Vallois [12]

\section{7) Biomecánica de la mano (agarre)}

El agarre de la mano son las fuerzas de contracción de los músculos de la mano y el antebrazo, estas se convierten en fuerza de agarre, así como las ventajas mecánicas del sistema músculo esquelético producen. Tenemos dos modelos biomecánicos que son: la curvatura que adoptan los tendones de los músculos a su paso por la muñeca produce diferentes fuerzas de rozamiento en función del radio de la curvatura. El otro modelo es la descomposición de los esfuerzos existentes en 
la inserción del ligamento, según el ángulo que forman los tendones de los músculos respecto de las falanges donde se insertan. Esto nos pueda determinar la fuerza [1].

El grosor de agarre recomendado varía entre ocho y 16 milímetros $(\mathrm{mm})$ para los desatornilladores y entre 13 y $30(\mathrm{~mm})$ para estiletes. El tamaño óptimo que varía según el tamaño de la mano, esta entre 55 y $65(\mathrm{~mm})$ para hombres y entre 50 y $60(\mathrm{~mm})$ para las mujeres. Las personas con manos pequeñas no deben realizar tareas repetitivas que impliquen agarres de fuerza con mangos de diámetros superior a $60 \mathrm{~mm}$ [13].

\section{B. Herramientas manuales}

Dentro de las herramientas clasificadas podemos mencionar las siguientes: que realizan tareas por propia fuerza: herramientas de corte, de apriete y de repercusión. Según el esfuerzo de la acción de la mano: torsión, aprehensión, tracción y empuje. Herramientas manuales motrices: eléctricas, aire comprimido, de combustión interna (gasolina) y de explosión.

En las herramientas de corte se recomienda mantener permanentemente los filos, ya que si no se hace se incrementa los esfuerzos necesario y más fatiga para el trabajador. Adicionalmente, están los mangos de agarre, existiendo dos tipos que condicionan la morfología y las dimensiones de los mangos. Estos son: el agarre de fuerza donde los dedos y el pulgar se comprimen alrededor de la herramienta para proporcionar la máxima superficie de contacto entre la palma de la mano y el objeto. Este agarre sirve para aplicar momentos o evitar que los objetos roten. El otro es el agarre de precisión, este utiliza los pequeños músculos de la mano que tienen mayor control nervioso pero son más delicados y se fatigan antes [1].

Las herramientas de dos mangos se pueden clasificar como los alicates, tijeras, pinzas y otras. La función principal de la fuerza coincide con la acción de acercar los dos mangos, aunque también se puede realizar conjuntamente otro tipo de esfuerzo. Estas herramientas tienen dos aspectos, por un lado la distancia entre las asas cuando se ejecuta el máximo esfuerzo. La fuerza de aprehensión depende de la abertura y el tamaño de la mano.
La otra característica es el diseño individual de los mangos, ya deben tener una pequeña curvatura que facilite a la adaptación de la mano y además deben estar recubiertos con materiales que favorezcan el contacto con la piel y una longitud adaptable para que apoye toda la mano [1].

Algunos instrumentos han sido diseñados con características físicas ergonómicas. Una herramienta ergonómica es aquella bien adecuada para la tarea que usted está realizando y apropiada a su mano sin provocarle posturas que causen fatiga muscular, presión de contacto dañino y otros riesgos de salud y seguridad. Si el trabajador utiliza una herramienta que se adapte y ajusta a su mano, o utiliza una herramienta que fue diseñada para otro uso, puede tener un riesgo de sufrir una lesión, provocando una enfermedad laboral [14].

\section{Metodología}

El estudio fue descriptivo-transversal, ya que se pretendió medir y describir las medidas antropométricas y la fuerza de prensión de la mano derecha. Los participantes fueron un grupo de trabajadores del sector de la construcción del género masculino, trabajadores entre 19 y 40 años. No se incluyeron aquellas personas que presentaban una patología funcional o estructural diagnosticada en el segmento superior (mano, muñeca, brazo y antebrazo) o que le impidan realizar tareas de prensión al momento de hacer las pruebas.

Los instrumentos que se utilizaron para el estudio fueron: una báscula, un vernier de ramas cortas de 0 a 200 milímetros, una cinta métrica. Para la evaluación antropométrica de la mano se seleccionaran las siguientes variables: la longitud de la mano, longitud palmar, anchura de la mano, anchura máxima de la mano, espesor de la mano, circunferencia de la mano, circunferencia máxima de la mano. Así mismo, se utilizó un dinamómetro hidráulico de mano para la medición de fuerza de prensión digito-palmar (medidor de la fuerza de la mano) con una fuerza de presión de hasta 90 kilogramos. Todos los datos recopilados se registraran en una ficha individual por trabajador, para después realizar el análisis a través de un paquete estadístico descriptivo, considerando la media, desviación estándar, distribuciones de frecuencia, máximas y mínimas registradas en la evaluación. 


\section{Resultados y CONCLUSIONES}

En el análisis de datos vamos a encontrar las más relevantes correlaciones de fuerza prensil y las medidas de la mano derecha de los trabajadores del sector de la construcción en la ciudad de Bogotá. Figuras 1 a 3.

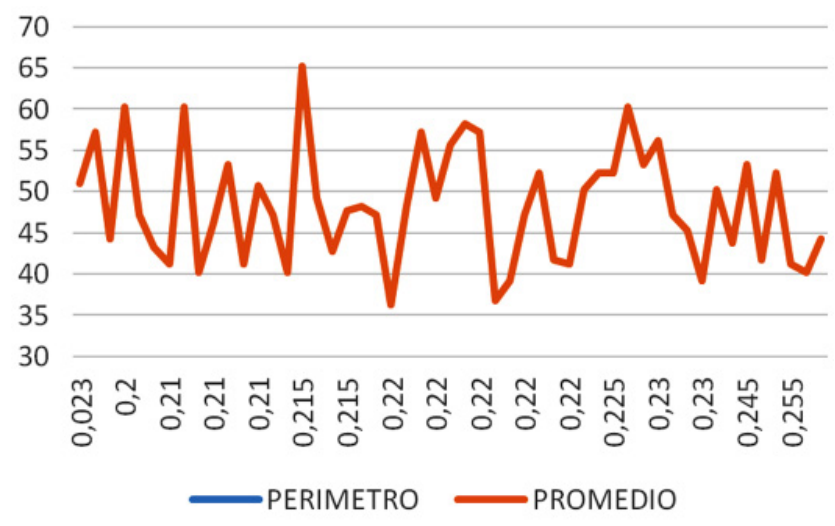

Fig. 1. Perímetro de la mano y fuerza. Fuente: Trabajo de campo.

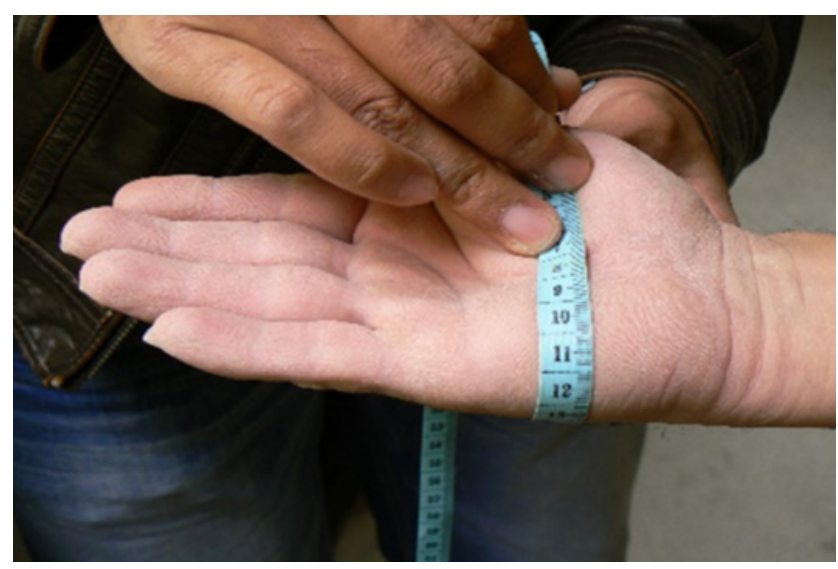

Fig. 2. Perímetro de la mano. Foto. Aldo Piñeda.

De esta gráfica donde se encuentra la relación de la fuerza de prensión de la mano derecha (eje Y) con el perímetro de la mano derecha (eje X), podemos analizar la siguiente: no hay una correlación fuerte entre la fuerza y el perímetro, debido a que los puntos tienen se encuentran muy dispersos. Podemos observar que a mayor perímetro de la mano, menor fuerza prensil ya que tenemos una pendiente negativa en el comportamiento de la gráfica. Podemos concluir que las personas con un perímetro menor aplicaron en el dinamómetro una mayor fuerza prensil. Figura 4.

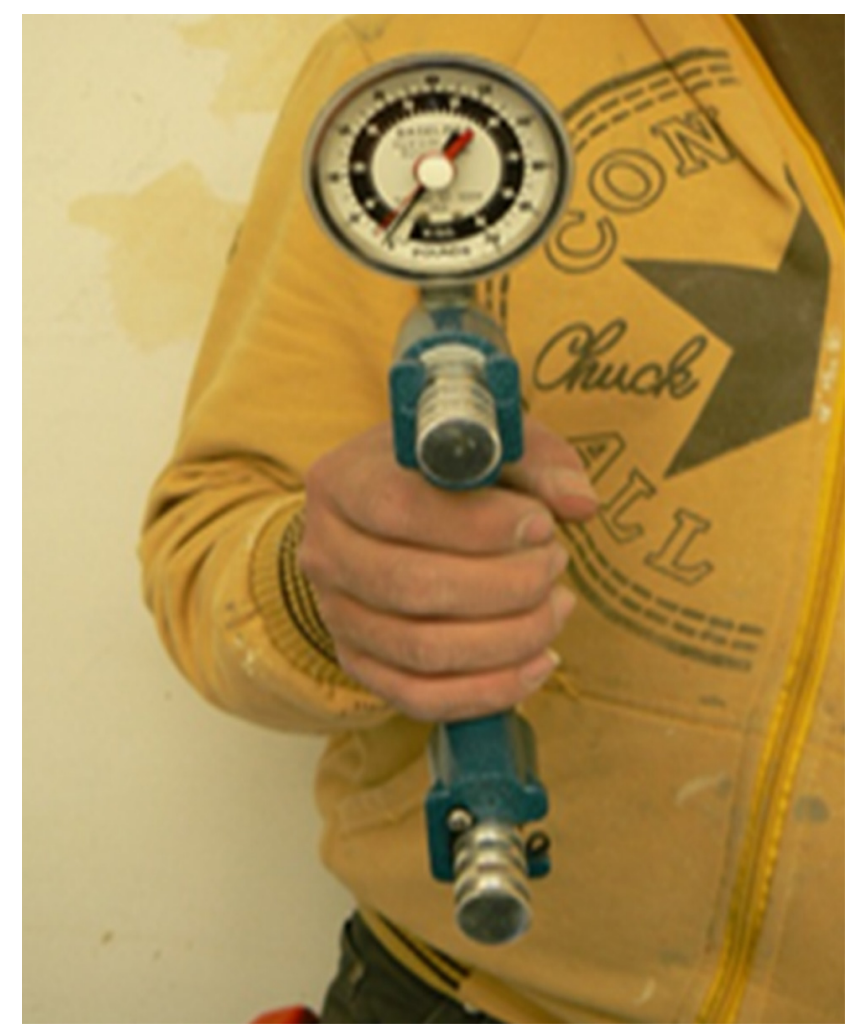

Fig. 3. Fuerza de la mano. Foto: Aldo Piñeda.



Fig. 4. Longitud de la mano y fuerza. Fuente: Trabajo de campo.

De esta grafica donde se encuentra la relación de la fuerza de prensión de la mano derecha (eje Y) con la longitud de la mano derecha (eje X), podemos siguiente análisis: podemos observar en la gráfica una correlación débil entre la fuerza prensil y la longitud de la mano derecha. Se puede determinar que sin importar la longitud de la mano derecha del trabajador se aplica una fuerza prensil en promedio es de $48 \mathrm{~kg} / \mathrm{cm}^{2}$, y la mayoría de los puntos obtenidos en la gráfica se encuentran muy cerca a este promedio. Figuras 5 y 6 . Vol. 3 / Núm. 5 / enero-junio de 2016; pág. 71-78 


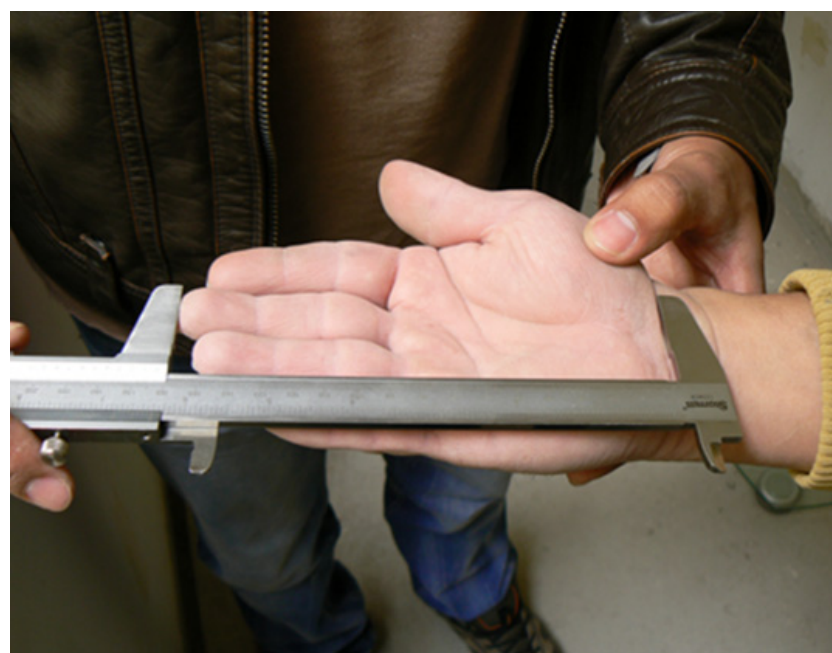

Fig. 5. Longitud de la mano. Foto: Aldo Piñeda.

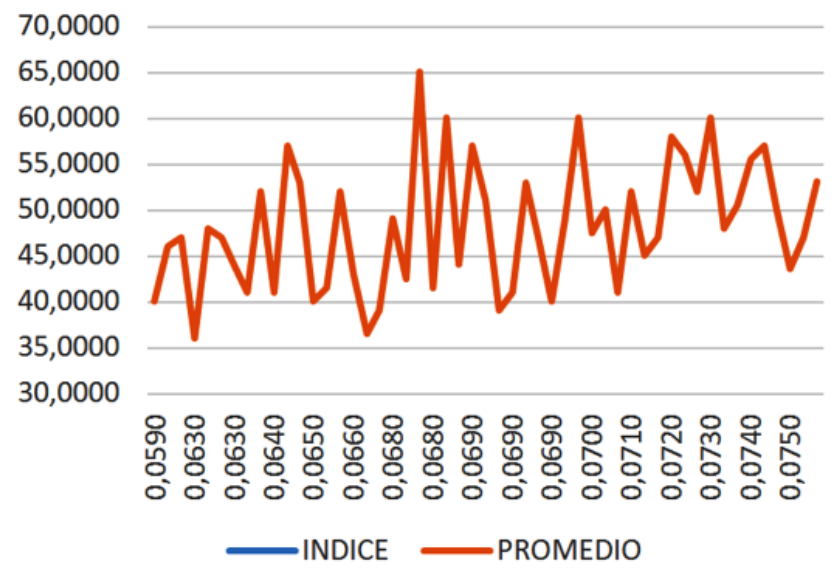

Fig. 7. Índice de mano derecha.

De esta gráfica donde se encuentra la relación de la fuerza de prensión de la mano derecha (eje Y) con el índice de la mano derecha (eje X), podemos analizar la siguiente: en la gráfica podemos observar que a mayor longitud del dedo índice de la mano mayor fuerza prensil puede aplicar el trabajador. La pendiente de la gráfica es positiva ya que a mayor longitud mayor fuerza prensil. Una persona con una medida superior a $68 \mathrm{~mm}$ puede tener una fuerza prensil superior a $50 \mathrm{~kg} / \mathrm{cm}^{2}$.

Analizando las gráficas encontramos que la mayor correlación entre medidas antropométricas de la mano y la fuerza están en el dedo índice y el dedo medio, se puede decir que a mayor dimensión de los dedos mayor fuerza prensil. De esta manera se identificaron los rangos antropométricos de la mano derecha para el diseño de herramientas manuales en el sector de la construcción.
La descripción de estas medidas y la fuerza prensil constituye para un futuro hacer una mayor cobertura de las medidas antropométricas y los aspectos de dinamometría para el sector de la construcción, así mismo para el diseño de uso manual ergonómico. Entre las herramientas que usan los trabajadores están: destornillador de pala y estrella, formon, pinzas, martillo, espátula, cepillo para madera, pistola de calafateo de tubo, mazo o chipole, lima y prensas.

Las recomendaciones para los trabajadores es la gestión del conocimiento y se debe poner en práctica los conceptos aprendidos, son los trabajadores quienes deben usar las nuevas herramientas para que disminuyan los riesgos laborales por la manipulación de herramientas no adaptadas para su mano.

En cuanto a la empresa, se deben diseñar estrategias pedagógicas para enseñar a los trabajadores el adecuado uso de herramientas manuales y su manipulación durante sus labores y para los fabricantes de herramientas manuales, mejorar el diseño de las herramientas para una mejor adaptación de las herramientas a las manos de los trabajadores y así mitigar algunos riesgos laborales para este sector de la construcción.

\section{REFERENCIAS}

[1] F. Farrer, G. Minaya, J. Niño, M. Ruiz, «Manual de Ergonomía.» MAPFRE. Madrid, España, 1995.

[2] E. Cerda, N. Cubillos, O. Medina, C. Rodriguez, «Ciencia \& Trabajo.» 2011.

[3] R. P. Mondelo, G. Enrique, J. Blasco, P. Barrau, Ergonomía 3. Diseño de puestos de trabajo. México, D.F.: Alfaomega, 2001.

[4] S. Schneider, «Musculoskeletal injuries in construction: Are they a problem?,» presented at the IEA, Proceedings of the 13th Triennial Congress of the International Ergonomics Association, Tampere, Finland., 1997, vol. 6, pp. 343-345.

[5] S. Schneider, P. Susi, Ergonomics and Construction: A review of potential hazards in new constructions. Am Ind Hyg Assoc J., 1994.

[6] M. Bohuslav, «Riegos para la salud en obras subterráneas.» OIT. Enciclopedia de Salud y Seguridad en el Trabajo, 2007.

[7] G. Lemasters, M. Aterrbury, A. Booth-Jones, A. Bhattacharya, N. Ollila-Glenn, C. Forrester, L. Forst, 
Prevalence of work related musculoskeletal disorders in active union carpenters. Occupational and Environmental Medicine. 1998.

[8] L. Arias, «Biomecánica y patrones funcionales de la mano. Bogotá, D.C.,» Universidad Nacional de Colombia. Revista de morfología., p. 15, 2012.

[9] A. Viladot A, y Colaboradores, «Lecciones Básicas de Biomecánica del aparato Locomotor.» Springer.

[10] M. Sanders, E. J. McCormick, Human Factors in Engineering and Design. Singapore: McGraw-Hill International, 1987.
[11] J. Faulhaber, «Crecimiento: Somatometría de la adolescencia.» México, D.F. Universidad Nacional Autónoma de México, 1989.

[12] H. Vallois, Anthropometric technique., vol. 6, 2 vols. USA: Current Anthropology, 1965.

[13] T. Fraser, Herramientas. Ergonomía, vol. 1. Dufrensne. OIT.: Editorial Chantal, 1998.

[14] Instituto Nacional de Salud y Seguridad Ocupacional, «Ergonomía fácil: Un guía para la selección de herramientas de mano no-energizadas.» Departamento de Relaciones Industriales. Cincinnati. USA, 2004. 